\title{
The humble case report: bottom of the evidence-based medical pyramid or the foundation of clinical research?
}

\author{
a \\ Darren Mylotte, MB, BCh, MD, Deputy Editor
}

During a recent EuroIntervention editorial board meeting, the members discussed the merits of publishing a small case series $(\mathrm{N}<4)$ reporting a potential problem with a frequently used device in the field of structural heart interventions. Board members in favour of publishing the manuscript spoke of the importance of disseminating original observations from day-to-day clinical practice and the impact these reports can have on our field. Those less inclined to publish this submission raised concerns about the validity of the observation, the small number of subjects included, the absence of a clear clinical impact, and the position of case reports in the hierarchy of medical evidence.

Although considered to rank among the lower echelons of the evidence-based medical pyramid, careful clinical observation disseminated as a humble case report or small series can stimulate more robust clinical research on a particular topic. But can a case report change the world?

At the American Heart Association annual meeting in Miami, November 1976, Dr Andreas Grüntzig first presented a small series of balloon dilatations of ligated canine coronary arteries using a double lumen catheter ${ }^{1}$. These experiments laid the foundation for the treatment of the first patient, 38-year-old Adolf Bachmann, on 16 September 1977 at the Universitätsspital in Zürich, Switzerland ${ }^{2}$. This single case report, brilliantly chronicled by Bernie Meier in this journal last year for the celebration of the 40-year anniversary of angioplasty ${ }^{3}$, changed the lives of Andreas Grüntzig and of his patient Adolf Bachmann, and has had an immeasurable impact on the lives of millions of patients undergoing interventional cardiology procedures.

A more contemporaneous example of a case series that altered the course of our specialty was published in The Lancet in 2004. Eugene McFadden and colleagues reported four cases of "late" first-generation drug-eluting stent (DES) thrombosis after the discontinuation of dual antiplatelet therapy ${ }^{4}$. The impact of this observation was immediately apparent, spawning the DES "firestorm" at the European Society of Cardiology meeting in Barcelona, 2006, and tempering the initial enthusiasm for DES technology. Although contemporary antiplatelet agents, periprocedural antithrombin therapy, and third-generation DES have greatly diminished the incidence of late stent thrombosis, the McFadden report highlighted important gaps in our understanding of the interactions between the patient, stent, polymer, drug, and implant technique that were rigorously investigated in the aftermath of his initial publication.

More recently, Colm Hanratty and Simon Walsh from the Royal Victoria Hospital, Belfast, published a case series of three patients in this journal. These authors described, for the first time, the phenomenon of longitudinal stent compression/deformation ${ }^{5}$. This original observation has been linked to stent thrombosis 
and a latent risk of restenosis, and has had a profound impact on interventional practice. Although stent deformation occurred with a variety of stent platforms, subsequent bench testing demonstrated certain DES to have higher structural integrity than others ${ }^{6}$. For the first time, clinicians started to select specific DES for ostial or left main interventions and for heavily calcified proximal stenoses. Moreover, this report brought about a renewed focus on careful guide catheter manipulation and stent deployment, provided yet another indication for procedural optimisation with intravascular imaging, and prompted the redesign of certain stent platforms. Almost seven years later, this case series has been cited $>70$ times.

Perhaps the most remarkable case report that I have read in recent years was published in the New England Journal of Medicine last month: "Self-Management of an Inferior ST-Segment Elevation Myocardial Infarction"' . These authors report the case of a 44-yearold nurse working at a remote nursing outpost in Western Australia who self-diagnosed an inferior ST-segment myocardial infarction complicated by complete heart block. As other medical personnel were not available, the patient self-cannulated both antecubital veins, self-prescribed appropriate medical therapy, sited defibrillator pads, and prepared bail-out antiarrhythmic drugs, before selfadministering thrombolysis. The symptoms and electrocardiogram settled post-lysis, and the Royal Flying Doctor Service transferred the patient to Perth for DES implantation the next day. The subject of the report, nurse Ryan Franks, did not have to fly the aeroplane!

In this edition of EuroIntervention, three unique Flashlights (case reports or series) are presented. The first from Odense, Denmark, reports a remarkable case of a 65 -year-old male presenting with out-of-hospital cardiac arrest who required 74 minutes of cardiopulmonary resuscitation, primary PCI to an acute right coronary occlusion, and circulatory support with biventricular assist devices (Impella ${ }^{\circledR}$ CP and RV; Abiomed, Danvers, MA, USA) ${ }^{8}$. Article, see page 2114

The patient was discharged home and remains well at one year. Will this case influence more liberal use of percutaneous biventricular support, even in seemingly futile situations?

A second Flashlight from the Mayo Clinic, Rochester, MN, USA, describes the first published case of balloon valvuloplasty of an acutely thrombosed mechanical valve (tricuspid position) ${ }^{9}$. This manoeuvre was successfully performed in a patient with progressive cardiogenic shock deemed unsuitable for either thrombolysis or surgery, and was associated with a favourable clinical outcome.

\section{Article, see page $\mathbf{2 1 2 2}$}

This report provides a potential treatment option for right-sided mechanical valve thrombosis (or stenosis?) in the very rare situation when thrombolysis or surgery is contraindicated.

Finally, Dr Liu and colleagues from Xi' an in China describe a novel minimally invasive technique to reduce left ventricular outflow tract obstruction in hypertrophic obstructive cardiomyopathy (HOCM) using percutaneous transapical intraseptal radiofrequency ablation ${ }^{10}$. This novel procedure was successfully performed in a series of five patients and could be a potential alternative to septal reduction surgery or alcohol septal ablation in HOCM. A larger clinical trial of this technique is planned.

Article, see page 2112

The current edition's Flashlights may not have such a far-reaching impact as Andreas Grüntzig's first angioplasty, but each makes an important contribution to the iterative understanding of our field. Case reports cannot have the impact of larger, more carefully designed controlled trials. They are at opposite ends of the medical evidence pyramid. Fundamentally, however, all medical research amounts to the principle of careful clinical observation. The humble case report is the foundation of this principle.

The Editorial Board of EuroIntervention is committed to publishing two to three high-quality basic science or clinical observation "Flashlights" per edition and welcomes interesting and educational submissions. Your case report might not change the world, but then again, it might.

\section{References}

1. Grüntzig A, Turina M, Schneider J. Experimental percutaneous dilatation of coronary artery stenosis (abstract). Circulation. 1976;54(Suppl):81.

2. Gruntzig A. Transluminal dilatation of coronary-artery stenosis. Lancet. 1978;1:263.

3. Meier B. His master's art, Andreas Gruntzig's approach to performing and teaching coronary angioplasty. EuroIntervention. 2017; 13:15-27.

4. McFadden EP, Stabile E, Regar E, Cheneau E, Ong AT, Kinnaird T, Suddath WO, Weissman NJ, Torguson R, Kent KM, Pichard AD, Satler LF, Waksman R, Serruys PW. Late thrombosis in drug-eluting coronary stents after discontinuation of antiplatelet therapy. Lancet. 2004;364:1519-21.

5. Hanratty CG, Walsh SJ. Longitudinal compression: a "new" complication with modern coronary stent platforms--time to think beyond deliverability? EuroIntervention. 2011;7:872-7.

6. Ormiston JA, Webber B, Webster MW. Stent longitudinal integrity bench insights into a clinical problem. JACC Cardiovasc Interv. 2011;4:1310-7.

7. Lee F, Maggiore P, Chung K. Self-Management of an Inferior ST-Segment Elevation Myocardial Infarction. $N$ Engl J Med. 2018;378:960-2.

8. Ellert J, Jensen MJ, Jensen LO, Møller JE. Percutaneous biventricular cardiac assist device in cardiogenic shock and refractory cardiac arrest. EuroIntervention. 2018;13:e2114-5.

9. Goel K, Gulati R, Bell MR. Thrombosed mechanical tricuspid valve: emergent valvuloplasty as rescue. EuroIntervention. 2018;13:e2122-3.

10. Liu L, Liu B, Li J, Zhang Y. Percutaneous transapical intraseptal radiofrequency ablation of hypertrophic obstructive cardiomyopathy: a novel mini-invasive treatment for reduction of outflow tract obstruction. EuroIntervention. 2018;13:e2112-3. 